\title{
Original Communication
}

\section{Parenteral Structured Triglyceride Emulsion Improves Nitrogen Balance and Is Cleared Faster from the Blood in Moderately Catabolic Patients*}

\author{
Joanna W. Kruimel, MD $†$; Ton H. Naber, $\mathrm{MD}, \mathrm{PhD} \dagger ;$ J. Adam van der Vliet, $\mathrm{MD}, \mathrm{PhD}$; \\ Claes Carneheim, PhD $\$$; Martijn B. Katan, PhD†; and Jan B. Jansen, MD, PhD $\dagger$ \\ From the $\uparrow$ Department of Gastroenterology and the $\ddagger$ Department of Surgery, University Medical Center \\ Nijmegen, Nijmegen, The Netherlands; and \$Fresenius-Kabi, Stockholm, Sweden
}

\begin{abstract}
Background: Most postoperative patients lose net protein mass, which reflects loss of muscle tissue and organ function. Perioperative parenteral nutrition may reduce the loss of protein, but in general, with conventional lipid emulsions a waste of protein still remains. Methods: We compared the effects on nitrogen balance of an emulsion containing structured triglycerides, a new type of synthesized triglycorides, with an emulsion of a physical mixture of medium- and long-chain triglycerides as part of parenteral foeding in moderately catabolic patients. The first 5 days after placomenti of an aortic prosthesis patients received total parontergl nutrition (TPN) providing $0.2 \mathrm{~g}$ of nitrogen per $\mathrm{kg}$ body woight per day; energy requirement was calculated utsing Harris and Benedict's equation, adding $300 \mathrm{kcal}$ per day for activity. Twelve patients were treated with the structured triglyceride emulsion and 13 patients with the emulsion of tho physical mixture of medium- and long-chain triglycorides. The design was a randomized, double-blind
\end{abstract}

After major surgery, most patients become catabolic and lose net protein mass, resulting in loss of muscle lissue and organ function. Improvement of protein retention is important for conservation of muscle mass and organ function. Perioperative parenteral nutrition may reduce the loss of protein ${ }^{1}$ and improve outcome in severely malnourished patients, ${ }^{2,3}$ but with existing regimes a considerable waste of protein still remains.

Lipid emulsions are an important component of total parenteral nutrition (TPN). They supply energy and essential fatty acids. Soybean oil is the usual source of fat in parenteral fat emulsions and consists of longchain triglycerides. Medium-chain triglycerides have been suggested as an alternative lipid source, because they may improve nitrogen balance, ${ }^{4,5}$ are metabolized more rapidly and stored less in tissues, ${ }^{6}$ and are oxi-

Rocoived for publication, February 3, 2000.

Accepted for publication, April 302001.

Correspondence: T.FI, Naber, Department of Gastroenterology, University Medical Center Nijmegen, Geert Grooteplein Zuid 8, $6500 \mathrm{HB}$

Nijmogen, The Netherlands. Electronic mail may be sent to T.Naber@gastro.azn.nl.

"WrThis study was presented at the A.S.P.E.N. Clinical Congress in 1997. Supported, in part, by a grant from Fresenius-Kabi. parallel study. Results: In the patients who completed the study, the mean cumulative nitrogen balance over the first 5 postoperative days was $-8 \pm 2 \mathrm{~g}$ in 10 patients on the structured triglyceride emulsion and $-21 \pm 4 \mathrm{~g}$ in 9 patients on the emulsion of the physical mixture of medium- and long-chain triglycerides; the mean difference was $13 \mathrm{~g}$ of nitrogen ( $95 \%$ confidence interval 4 to $22, p=.015$ ) in favor of the structured triglyceride emulsion. On the first postoperative day serum triglyceride and plasma medium-chain free fatty acid levels increased less during infusion of the structured triglyceride emulsion than with the physical mixture emulsion. Conclusions: The parenteral structured triglyceride emulsion improves the nitrogen balance and is cleared faster from the blood, compared with the emulsion of the physical mixture of medium- and long-chain triglycerides, in moderately catabolic patients. (Journal of Parenteral and Enteral Nutrition 25:237-244, 2001)

dized mainly independently of carnitine. ${ }^{6}$ However, high plasma levels of medium-chain free fatty acids, the metabolic product of medium-chain triglycerides, may cause adverse reactions, such as metabolic acidosis due to higher production of ketone bodies ${ }^{7}$ and increased energy expenditure. ${ }^{8}$ In dogs, high plasma medium-chain free fatty acid levels caused serious neurologic toxicity. ${ }^{9}$ To reduce the amount of mediumchain triglycerides and to provide the essential longchain fatty acids, the medium-chain triglycerides are administered together with long-chain triglycerides, as a physical mixture.

To improve the safety of medium-chain triglycerides, so-called structured triglycerides were synthesized. Structured lipids are produced by hydrolysis of soybean oil and coconut oil. Re-esterification results in structured triglycerides with medium- and long-chain fatty acids at randomly attached to positions within the same glycerol molecule. An emulsion containing these structured triglycerides caused lower plasma levels of medium-chain free fatty acids and was cleared faster from the blood compared with an emulsion of a physical mixture of medium-chain and long-chain triglycerides, in healthy volunteers. ${ }^{10,11}$ 
The question has been asked if this structured triglyceride emulsion improves nitrogen balance. In animals, parenteral administration of emulsions of structured triglycerides leads to improved nitrogen balance, ${ }^{12-14}$ increased hepatic protein synthesis ${ }^{13,14}$ and serum albumin levels, ${ }^{13}$ and decreased leucine oxidation ${ }^{12,14}$ compared with emulsions of a physical mixture of medium- and long-chain triglycerides.

The predefined objectives of the present clinical trial were to study, in postoperative, moderately catabolic patients, the influence on nitrogen balance and the safety and tolerance of intravenous (IV)-infused structured triglycerides (Structolipid) compared with that of a physical mixture of medium- and long-chain triglycerides (Lipofundin MCT/LCT 20\%). The structured triglyceride emulsion has been compared with an emulsion of long-chain triglycerides, proven to be as safe and well tolerated, ${ }^{15}$ and associated with increased whole body fat oxidation in postoperative patients. ${ }^{16}$

\section{MATERIALS AND METHODS}

\section{Design}

The design was a randomized, double-blind, parallel study. We excluded some important confounders. We chose for a homogeneous and stable group of patients (only elective surgery), and enteral nutrition was not permitted until the end of the study period. We included patients operated for placement of an aortic tube or bifurcation prosthesis because of an abdominal aortic aneurysm or atherosclerotic obstruction. Such patients make a homogeneous group, are moderately catabolic, ${ }^{17}$ and may require postoperative parenteral nutrition. We chose to administer parenteral nutrition in all of these patients, although in clinical practice not all of these patients would need parenteral nutrition. Probably some patients, nutritional needs could be reached by tube feeding in the duodenum or jejunum.

We calculated the daily cumulative nitrogen balance for those patients who completed the 5-day study period. We assessed safety and tolerance in accordance with standard clinical practice. Serum levels of triglycerides and plasma levels of medium-chain free fatty acids were measured as an estimate of the clearance rate of the lipid emulsions from the blood. IV-administered triglycerides are hydrolyzed by lipoprotein lipase, producing long-chain and medium-chain free fatty acids.

\section{Patients}

The study was approved by the Human Ethics Committee of the University Hospital of Nijmegen, The Netherlands. Over a period of 20 months, 61 patients were admitted to the Department of Surgery for elective surgery. Placement of an aortic tube or bifurcation prosthesis because of an abdominal aortic aneurysm or atherosclerotic obstruction was performed. Thirty-four patients were excluded because of hypertriglyceridemia (9), treatment with lipid lowering-drugs (8), not giving written consent (7), treatment with corticosteroid hormones (5), renal disease (3), diabetes mellitus
(2), age above 80 years (1), severe overweight (1), or fluid restriction (1). Three patients fulfilled 2 exclusion criteria.

Twenty-seven patients were informed by verbal and written consent, after which they were randomized. In none of the patients was significant weight loss observed before the hospitalization. Preoperatively the patients were normally active. In all of these patients elective surgery was performed. Two patients dropped out before treatment started: 1 changed her decision to participate, and 1 suffered a myocardial infarction after inclusion in the study. We used a table with random numbers to assign patients to receive either the structured triglyceride emulsion $(n=12)$ or the emulsion of the physical mixture of medium- and longchain triglycerides $(n=13)$.

Four patients were withdrawn after treatment had started for reasons not related to the lipid emulsions: Adult Respiratory Distress Syndrome, aspiration of stomach contents, ventricular fibrillation, and thrombosis in the subclavian vein.

Postoperatively these patients were not on a respiratory ventilator during the nitrogen balance study, and patients were mobilized as soon as possible. After 5 days, when the study was completed, oral intake was started if possible.

Before nitrogen excretion was measured, and still blinded to treatment, one patient was excluded from calculation of the nitrogen balance. He was treated with the physical mixture. The reason for exclusion of this patient was extensive muscle breakdown due to muscle ischemia and compression, which is known to produce high nitrogen excretion. In one patient treated with the structured triglyceride, day 4 emulsion urine was lost. This patient was also excluded from calculation of the nitrogen balance. Therefore nitrogen balance was calculated for 10 patients treated with the structured triglyceride emulsion and for 9 patients treated with the emulsion of the physical mixture who completed the 5-day study period.

\section{Treatment}

In all patients parenteral nutrition was started with an administration rate according to nutritional need. Patients were treated with a structured triglyceride emulsion (Structolipid, Fresenius-Kabi, Sweden) or an emulsion of a physical mixture of medium- and longchain triglycerides (Lipofundin MCT/LCT 20\%, B. Braun Melsungen AG, Germany) (Table I). Figure 1 shows the molecular structure of MCT, LCT, and structured triglycerides. Structured triglycerides are produced by hydrolysis of MCT and LCT in glycerol and free fatty acids and subsequent at random re-esterification of glycerol with free fatty acids, 1,3-specific lipase is not used. Both patient groups received an equal dose of lipid emulsion in weight with an equal energy content, because we chose to compare the emulsions under energetically equivalent conditions. The patients, treated with the physical mixture-emulsion, received 1.08 times more triglycerides and 1.25 times more medium-chain fatty acids, both on a molar base.

The patients were treated with TPN for the first 5 days after surgery. We calculated energy requirement 
TABLE I

Composition and characteristics of the structured triglyceride emulsion and of the emulsion of the physical mixture of mediumand long-chain triglycerides

\begin{tabular}{|c|c|c|}
\hline Composition and characteristics & $\begin{array}{c}\text { Structured } \\
\text { triglyceride } \\
\text { emulsion } \\
\end{array}$ & $\begin{array}{l}\text { Physical } \\
\text { mixture } \\
\text { emulsion }\end{array}$ \\
\hline Structured triglycerides $(\mathrm{g} / \mathrm{L})$ & 200 & \\
\hline Soy bean oil $(\mathrm{g} / \mathrm{L})$. & & 100 \\
\hline Medium-chain triglycerides $(\mathrm{g} / \mathrm{L}$ ) & & 100 \\
\hline Mean molecular weight of & & \\
\hline $\begin{array}{l}\text { triglycerides } \\
\text { Fractionsted }\end{array}$ & 683 & 634 \\
\hline Fractionated egg phospholipids (g/L) & 12 & 12 \\
\hline Glycerol (USP) (g/L) & 22.5 & 25.0 \\
\hline Water for injection ad (mL) & 1000 & 1000 \\
\hline $\mathrm{pH}$ & 8 & $6.5-8.5$ \\
\hline Osmolality (mosm/kg water) & 350 & 380 \\
\hline Energy content (kcal/L) & 1960 & 1936 \\
\hline \multicolumn{3}{|l|}{ Fatly acid composition, \% by weight } \\
\hline Caprylic acid (C8:0) & 27 & 26 \\
\hline Capric neid (C,10:0) & 10 & 20 \\
\hline Palmitic acid (C16:0) & 7 & 7 \\
\hline Stonric acid (C18:0) & 3 & 2.5 \\
\hline Olsic acid (C18:1) & 13 & 13 \\
\hline Linoleic acid (C18:2w6) & 33 & 27 \\
\hline Alpha-linolenic acid (C18:3w3) & 5 & 3.5 \\
\hline Othor & 2 & 1 \\
\hline
\end{tabular}

in kcal/24 h using Harris and Benedict's equation [males: $66.47+13.75 \times$ weight $(\mathrm{kg})+5.0$ height $(\mathrm{cm})-6.76 \times$ age (years); females: $655.10 \times 9.56 \times$ weight $(\mathrm{kg})+1.85 \times$ height $(\mathrm{cm})-4.68 \times$ age (years)] and added $300 \mathrm{kcal} / 24 \mathrm{~h}$ for activity. We gave $0.2 \mathrm{~g}$ of nitrogen/kg body weight per $24 \mathrm{~h}$ (Vamin 18, Fresenius-Kabi, Sweden), which is in accordance to the nutritional needs of this group of patients. ${ }^{18}$ Twothirds of the non-protein energy or $53 \%$ of total energy was given as carbohydrates (glucose $40 \%$ ), one-third or $26 \%$ of total energy as lipid emulsion, and $21 \%$ of total energy as amino acids. Amino acids and carbohydrates wore given daily over 24 hours, throughout the 5-day postoperative period. The lipid emulsions were administered separately, because stability studies of mixtures of the structured triglyceride emulsion, and amino acids and carbohydrates were not available. The lipid emulsions were administered daily from $10 \mathrm{AM}$ to $4 \mathrm{I} M$, because lipids could disturb the laboratory assessments and baseline blood samples were taken at $8 \mathrm{AM}$. This intermittent administration of lipid emulsions allowed us to study the kinetics of the lipid emulsions. All parenteral nutrition was administered through one lumen of a double-lumen subclavian catheter. IV medication was given separately through the other lumen of the subclavian catheter or via a periphoral catheter. Amino acids, carbohydrates and lipid emulsion were given at constant rates using 3 separate infusion pumps (Terufusion STC-503, Terumo, The Netherlands). Oral intake of nutrients was not allowed during the study period.

\section{Assessments}

The following baseline characteristics of the patients were determined: sex, race, age, nutritional status, duration of surgery, blood loss during surgery, and Acute Physiololgy and Chronic Health Evaluation
(APACHE) II score. ${ }^{19}$ Nutritional status was assessed by measuring height and weight, and calculating body mass index [weight per square height]. Furthermore, skinfold thickness according to Durnin and Womersley ${ }^{20}$ was measured at 4 sites (biceps, triceps, subscapular and supra-iliac), giving the body fat content of the patient; the percentage of ideal body fat content was calculated. Also, width of condyle of femur was measured according to de Wijn, ${ }^{21}$ giving the ideal weight of the patient; the body weight was measured and the percentage of ideal body weight was calculated.

Blood pressure, heart rate, respiratory frequency, body temperature, and adverse events of each patient were monitored, each postoperative day at $8 \mathrm{AM}$ and 4 PM. The patients especially were monitored for infectious complications, allergic reactions, nausea, chills, and neurologic symptoms. In addition, the APACHE II score was evaluated on days 1,3 , and 6 at $8 \mathrm{AM}$.

We calculated the nitrogen balance per 24 hours from the amounts of nitrogen administered parenterally and the nitrogen excreted in the urine. We assumed a daily loss of $2 \mathrm{~g}$ of nitrogen via other routes. ${ }^{18}$ During the first 5 days postoperatively nitrogen was only provided by the parenteral nutrition because oral intake of nutrients was not allowed. Under close supervision urine was collected daily and pooled in 24-hour aliquots from 6 AM until 6 AM the following day, and the urine was transported every morning to the laboratory at 8 AM. Urinary nitrogen content was analyzed according to Kjeldahl. ${ }^{22}$

Serum levels of triglycerides and plasma levels of medium-chain free fatty acids were measured on the first and fifth day after surgery before and at the end of lipid infusion, ie, at $8 \mathrm{AM}$ and $4 \mathrm{PM}$; one more sample was taken at the end of the study, on the sixth day at 8 AM. Serum triglycerides were measured by the Böhringer Mannheim Kit for triglyceride analysis without free glycerol. Plasma medium-chain free fatty acids were analyzed according to Tsuchiya et al. ${ }^{23}$ The free fatty acids were separated by reversed-phase highperformance liquid chromatography (HPLC) on a C18

\section{LCT Structured triglycerides MCT}

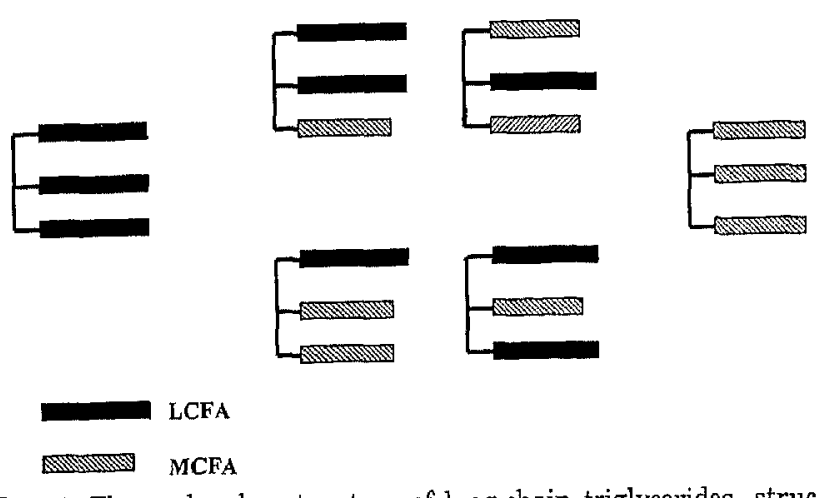

Fig. 1. The molecular structure of long-chain triglycerides, structured triglycerides, and medium-chain triglycerides. 
TABLE II

Baseline characteristics of patients treated with the structured triglyceride emulsion or with the physical mixture emulsion*

\begin{tabular}{|c|c|c|}
\hline Baseline characteristics & $\begin{array}{l}\text { Structured } \\
\text { triglyceride } \\
\text { emulsion }\end{array}$ & $\begin{array}{l}\text { Physical } \\
\text { mixture } \\
\text { emulsion }\end{array}$ \\
\hline Men/women (number) & $10 / 2$ & $10 / 3$ \\
\hline Caucasian/noncaucasian (number) & $12 / 0$ & $12 / 1$ \\
\hline Age (years) & $67 \pm 6$ & $69 \pm 7$ \\
\hline Height (m) & $1.68 \pm 0.09$ & $1.66 \pm 0.09$ \\
\hline Weight (kg) & $73 \pm 10$ & $69 \pm 13$ \\
\hline Body Mass Index $\left(\mathrm{kg} / \mathrm{m}^{2}\right)$ & $26 \pm 3$ & $25 \pm 3$ \\
\hline$\%$ of ideal body fat content & $106 \pm 5$ & $105 \pm$ \\
\hline$\%$ of ideal body weight & $108 \pm 12$ & $106 \pm 9$ \\
\hline Duration of surgery (min) & $150 \pm 70$ & $160 \pm 40$ \\
\hline Blood loss during surgery (mL) & $1420 \pm 680$ & $2130 \pm 880$ \\
\hline APACHE II score & $7 \pm 1$ & $7 \pm 1$ \\
\hline
\end{tabular}

Values are numbers or means $\pm \mathrm{SD}$.

column after a derivatization reaction with a fluorescent reagent, 4-bromomethyl-7-acetoxycoumarin. The free fatty acid derivatives were detected by fluorescence detection.

Blood hemoglobin, white blood cell count with differential count and platelet count, serum sodium, potassium, urea, creatinine, total bilirubin, alkaline phosphatase, aspartate aminotransferase, glucose, and $\beta$-hydroxybutyrate were measured before surgery, at 8 AM on day 1 before the start of parenteral nutrition, and at $8 \mathrm{AM}$ on days 3 and 6 . Beta-hydroxybutyrate was determined on a centrifugal analyser (Multistat III) ${ }^{24}$

\section{Statistical analysis}

The primary endpoint for comparison between the groups was the daily cumulative nitrogen balance calculated for those patients who completed the 5-day study period. Two secondary end points were tested: changes in serum levels of triglycerides and changes in plasma levels of medium-chain free fatty acids. All results were expressed as mean \pm SEM. Changes in the structured triglyceride-emulsion group were compared with changes in the physical mixture-emulsion group, using Student's $t$ test, and the 95\% confidence level was calculated.

\section{RESULTS}

Baseline characteristics of the two groups were similar with respect to sex, race, age, nutritional status, duration of surgery, and APACHE II score. After treatment had started, 6 patients dropped out for calculation of the nitrogen balance over the first 5 days. Baseline characteristics of the 2 groups remained similar when these 6 patients were excluded. Blood loss during surgery was a little smaller in the structured triglyceride-emulsion group $(1420 \pm 680 \mathrm{~mL})$ than in the group treated with the emulsion of the physical mixture of medium- and long-chain triglycerides (2130 \pm $880 \mathrm{~mL}$ ) (Table II). The difference in blood loss did not influence the nitrogen balance study, because the nitrogen balance started the day after surgery.

The mean cumulative nitrogen balance was calculated for those patients who completed the 5-day study period. The mean cumulative nitrogen balances of the 2 study groups became significantly different on the fourth postoperative day: $-5 \pm 2 \mathrm{~g}$ of nitrogen for the 10 patients receiving the structured triglyceride emulsion and $-16 \pm 4 \mathrm{~g}$ of nitrogen for the 9 patients receiving the emulsion of the physical mixture of medium- and long-chain triglycerides. The mean difference in cumulative nitrogen balance over the 4 day-period was 11. g of nitrogen ( $95 \%$ confidence interval 2 to 19 , $p=.02$ ). After 5 days the mean cumulative nitrogen balance was $-8 \pm 2 \mathrm{~g}$ for the structured triglyceride emulsion and $-21 \pm 4 \mathrm{~g}$ for the emulsion of the physical mixture; the nitrogen balance was thus $13 \mathrm{~g}$ less negative for the structured triglyceride emulsion than for the emulsion of the physical mixture (95\% confidence interval 4 to $22, p=.015$ ) (Table III, Fig. 2a and $2 \mathrm{~b}$ ).

On the first postoperative day, serum levels of triglycerides and plasma levels of medium-chain free fatty acids increased less during the 6 hours of lipid infusion with the structured triglyceride emulsion compared

TABLE III

Cumulative nitrogen balance over the first five days after surgery for 10 patients treated with the structured triglyceride enmlsion and 9 patients treated with the physical mixture emulsion, who completed the five-day nitrogen balance

\begin{tabular}{|c|c|c|c|c|c|}
\hline Structured triglyceride emulsion & Day 1 & Day 2 & Duy 3 & Day 4 . & Day 5 \\
\hline $\begin{array}{l}\text { Nitrogen parenterally } \\
\text { administered (g) }\end{array}$ & $+15 \pm 1$ & $+30 \pm 1$ & $+45 \pm 1$ & $+60 \pm 2$ & $-74 \pm 2$ \\
\hline Urinary nitrogen excretion (g) & $-14 \pm 1$ & $-30 \pm 2$ & $-44 \pm 3$ & $-57 \pm 3$ & $-72 \pm 4$ \\
\hline $\begin{array}{l}\text { Assumed nitrogen loss via other } \\
\text { routes }(\mathrm{g})\end{array}$ & -2 & -4 & -6 & -8 & -10 \\
\hline Cumulative nitrogen balance (g) & $-1 \pm 1$ & $-4 \pm 1$ & $-5 \pm 2$ & $-5 \pm 2^{*}$ & $-8 \pm 2^{: * * *}$ \\
\hline Physical mixture emulsion & Day 1 & Day 2 & Day 3 & Day 4 & Day 5 \\
\hline $\begin{array}{l}\text { Nitrogen parenterally } \\
\text { administered }(g)\end{array}$ & $+14 \pm 1$ & $+27 \pm 2$ & $+41 \pm 2$ & $+55 \pm 3$ & $+68+4$ \\
\hline Urinary nitrogen excretion $(\mathrm{g})$ & $-14 \pm 1$ & $-31 \pm 3$ & $-46 \pm 5$ & $-63 \pm 6$ & $-79 \pm 7$ \\
\hline Assumed nitrogen loss via other & & & & & \\
\hline $\begin{array}{l}\text { routes }(\mathrm{g}) \\
\text { Cumulative nitrogen balance }(\mathrm{g})\end{array}$ & $\begin{array}{c}-2 \\
-2 \pm 1\end{array}$ & $\begin{array}{l}-4 \\
-8 \pm 1\end{array}$ & $\begin{array}{c}-6 \\
-11 \pm 3\end{array}$ & $\begin{array}{c}-8 \\
-16 \pm 4 *\end{array}$ & $\begin{array}{c}-10 \\
-21 \pm 4 k * *\end{array}$ \\
\hline
\end{tabular}

Values are means \pm SEM.

"Mean difference $11 \mathrm{~g}$ (95\% confidence interval $2 ; 19, p=0.02$ )

*⿻: Mean difference $13 \mathrm{~g}$ ( $95 \%$ confidence interval $4 ; 22, p=0.015$ ). 

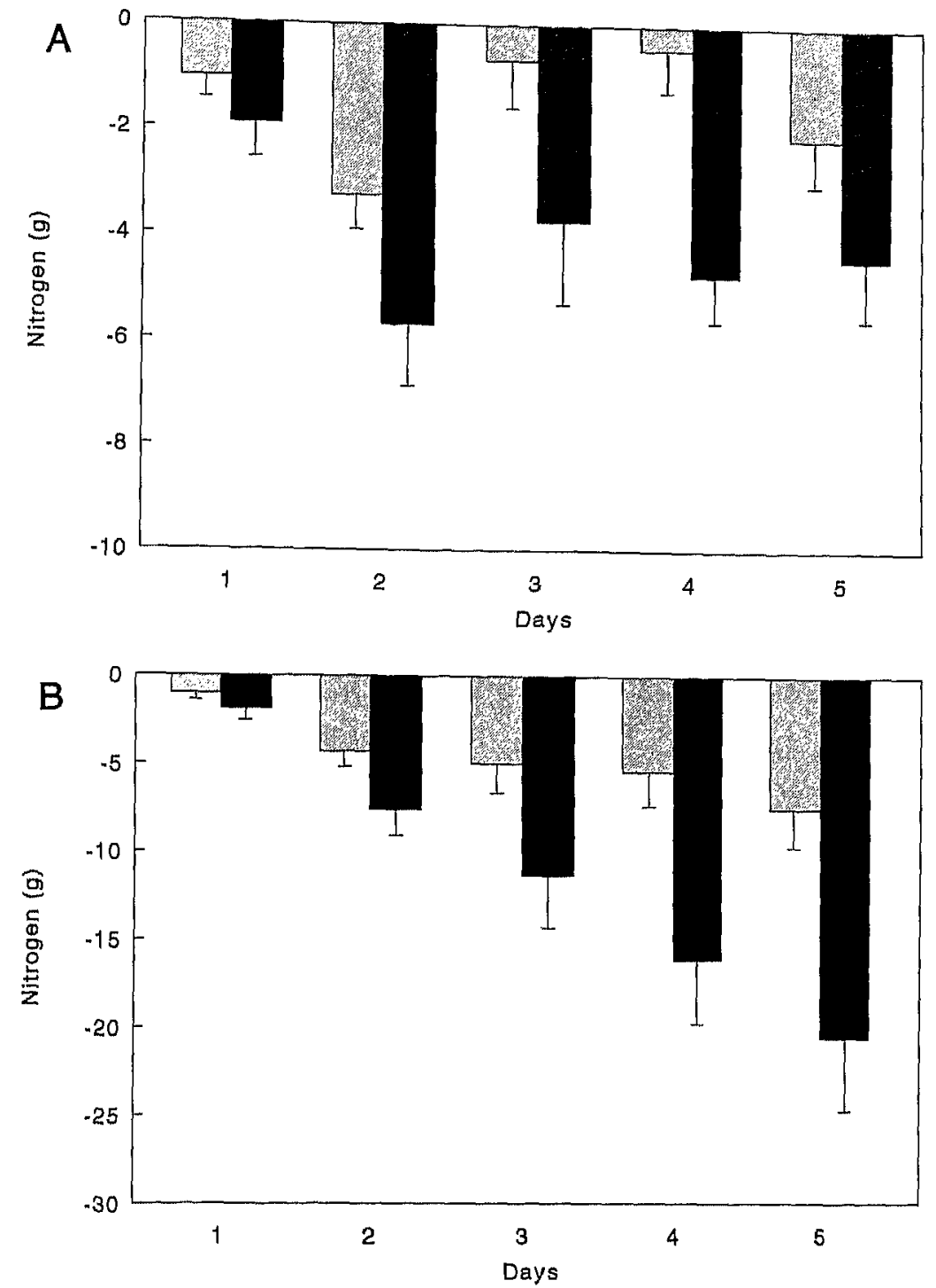

Fin, 2. (a) Daily nitrogen balances and (b) cumulative nitrogen balances over the first five days after surgery for 10 patients treated with the structurod triglyceride emulsion (hatched bars) and 9 patients treated with the physical mixture emulsion (solid bars), who completed the 5xelay mitrogen balance. Error bars indicate SEM.

with the physical mixture of medium- and long-chain triglycerides (the difference in change for triglycerides between treatment with the structured triglyceride emulsion and the physical mixture emulsion was 1.10 $\mathrm{mmol} / \mathrm{L}, 95 \%$ confidence interval 0.70 to $1.50, p<$ .00005 , and for medium-chain free fatty acids 145 $\mu \mathrm{mol} / \mathrm{L}, 95 \%$ confidence interval 99 to $161, p=.00001$, Table IV). These differences were higher than expected from the difference in emulsion composition on a molar base. Both groups received an equal dose of lipid emulsion in weight; thus, the physical mixture-emulsion group received 1.08 times more triglycerides and 1.25 times more medium-chain fatty acids, both on a molar base. However, serum triglycerides and plasma medium-chain free fatty acids concentrations increased 2 times more with the physical mixture-emulsion group.

\section{TABLE IV}

Changes in mean serum levels of triglycerides (mmol/L) and plasma levels of meditum-chain free fatty acids (MCFFA, $\mu$ mol/L) on day I post-operatively, from 8 AM, before the start of the lipid infusion, until 4 PM; the end of lipid infusion

\begin{tabular}{|c|c|c|c|}
\hline Time & Variables & Structured triglyceride emulsion & Physical mixture emulsion \\
\hline Day $1,8 \mathrm{AM}$ & $\begin{array}{l}\text { triglycerides } \\
\text { MCFFA }\end{array}$ & $\begin{aligned} 0.86 & \pm 0.07 \\
12 & \pm 4\end{aligned}$ & $\begin{array}{l}0.74 \pm 0.07 \\
7 \pm 3\end{array}$ \\
\hline Day $1,4 \mathrm{PM}$ & triglycerides & $1.44 \pm 0.12$ & $2.42 \pm 0.16$ \\
\hline Change day $1,4, P M-8$ AM & $\begin{array}{l}\text { MCFFA } \\
\text { triglycerides } \\
\text { MCFFA }\end{array}$ & $\begin{array}{c}149 \pm 13 \\
0.58 \pm 0.10 \\
137 \pm 14\end{array}$ & $\begin{array}{l}289 \pm 18 \\
1.68 \pm 0.17^{*} \\
282 \pm 18^{* * * *}\end{array}$ \\
\hline
\end{tabular}

SValues are means \pm SEM. 
TABLES $\mathrm{V}$

Changes in mean serum levels of triglycerides (mmol/L) and plasma levels of medium-chain free fatty acids (MCFFA, $\mu$ mol/L) on day 5 post-operatively, from $8 \mathrm{AM}$, before the start of the libid infusion, until $4 \mathrm{PM}$, at the end of lipid infusion

\begin{tabular}{|c|c|c|c|}
\hline Time & Variables & Struetured triglyecrides emulsion & Physical mixture emulsion \\
\hline Day 5, $8 \mathrm{AM}$ & $\begin{array}{l}\text { triglycerides } \\
\text { MCFFA }\end{array}$ & $\begin{aligned} & 1.12 \pm 0.20 \\
& 11 \pm 3\end{aligned}$ & $\begin{array}{l}1.10 \pm 0.04 \\
4 \pm 3\end{array}$ \\
\hline Day 5, 4 PM & triglycerides & $1.99 \pm 0.24$ & $2.24 \pm 0.17$ \\
\hline Change day 5, $4 \mathrm{PM}-8 \mathrm{AM}$ & $\begin{array}{l}\text { MCFFA } \\
\text { triglycerides } \\
\text { MCFFA }\end{array}$ & $\begin{array}{l}168 \pm 25 \\
0.87 \pm 0.17 \\
157 \pm 22\end{array}$ & $\begin{aligned} 231 & \pm 25 \\
1.14 & \pm 0.16, \mathrm{~ns} \\
227 & \pm 26, \mathrm{ng}\end{aligned}$ \\
\hline
\end{tabular}

§Values are means \pm SEM.

On the fifth postoperative day after infusion of the lipid emulsions, changes in serum triglycerides and plasma medium-chain free fatty acids concentrations were, although lower in the group receiving the structured triglyceride emulsion, not significantly different for the 2 patient groups (triglycerides $0.27 \mathrm{mmol} / \mathrm{L}, 95 \%$ confidence interval -0.19 to 0.73 ; medium-chain free fatty acids $70 \mu \mathrm{mol} / \mathrm{L}, 95 \%$ confidence interval 2 to $138, p=$ .07. Table V).

Adverse events, especially infectious complications, blood pressure, heart rate, respiratory frequency, body temperature, and APACHE II score did not show significant differences between both groups. Blood hemoglobin, white blood cell count with differential count and platelet count, serum sodium, potassium, urea, creatinine, total bilirubin, alkaline phosphatase, aspartate aminotransferase glucose, and $\beta$-hydroxybutyrate were also similar in both groups at 8 AM on days 1,3 , and 6 .

\section{DISCUSSION}

Nitrogen balance was measured to compare the efficacy of an emulsion containing "structured" triglycerides-ie, triglycerides having medium-chain and longchain fatty acids within the same molecule-and an emulsion of a physical mixture of medium-chain and long-chain triglycerides. A less negative nitrogen balance indicates conservation of protein mass, including muscle mass. Nitrogen balance studies are widely used as an index of effectiveness of nutrition support ${ }^{18}$; a better index is not yet available. ${ }^{25}$

An emulsion of a physical mixture of medium- and long-chain triglycerides is known to improve nitrogen balance in patients compared with long-chain triglyceride emulsions. ${ }^{4,5}$ We found that parenteral administration of a "structured" triglyceride emulsion improved nitrogen balance in patients after a large vascular operation compared with an emulsion of a physical mixture of medium- and long-chain triglycerides. Daily urinary nitrogen excretion was higher in the physical mixture-emulsion group, starting from the second day after surgery (Table III, Fig. 2a and 2b). The mean difference in nitrogen balance between the 2 treatment groups was $13 \mathrm{~g}$ of nitrogen in 5 days in favor of the structured triglyceride emulsion, which means that $80 \mathrm{~g}$ of protein or-in case this protein was completely derived from muscle-approximately $500 \mathrm{~g}$ of muscle mass was saved over 5 days by using the structured triglyceride emulsion. The daily amount of nitrogen administered to the patients was a little higher in the structured triglyceride-emulsion group, but based on the weight of the patients and calculated as $0.2 \mathrm{~g}$ of nitrogen $/ \mathrm{kg}$ body weight per $24 \mathrm{~h}$. Indeed, the weight of the patients in the structured triglyceride-emulsion group was not significantly higher than the weight of the patients in the physical mixtureemulsion group. Patients with a higher weight have in a steady state condition a higher nitrogen loss. We found that the patients in the structured triglycerideemulsion group with a higher weight had a lower nitrogen loss, indicating that the nitrogen balance is improved by the treatment with the structured triglyceride emulsion. The difference in blood loss during surgery between the 2 groups could not explain the difference in nitrogen balance because it was too small to effect the calculations. Moreover, the nitrogen balance study started the day after surgery. Our results agree with findings in laboratory animals: structured triglyceride emulsions improved protein retention compared with emulsions of a physical mixture of mediumchain and long-chain triglycerides. ${ }^{12 \cdots 1}$

We also studied serum triglycerides and plasma medium-chain free fatty acicls and observed that the levels of serum triglycerides and plasma mediumchain free fatty acids increased loss cluring infusion of the structured triglycorido emalsion than with the physical mixture emulsion. Both groups roceived an equal dose of lipid emulsion in weight; thus, tho physical mixture-emulsion group received 1.08 times more triglycerides and 1.25 times moro medium-chain fatty acids, both on a molar base. However, this does not explain that serum triglycorides an medium-chain free fatty acids incroased 2 timos more with the physical mixture-emulsion group. Tho smaller incroase of serum triglycerides and plasma medium-chain free fatty acids during infusion of the structured triglyceride emulsion may be due to a faster use of the structured triglycerides than of the triglycerides in the physical mixture emulsion. On day 5 the differences between the 2 lipid emulsions wero not significant, possibly because patients wero loss stressed on day 5 than on day 1. Another explanation to the docroase in changes of serum triglyceride levels on day 5 may be due to the increase in basal levels. This increase in basal levels is most likely due to a too short infusion free period, so the triglycerides were not fully romoved from the circulation at the sampling time. The lack of difference in changes in plasma medium-chain frec fatty acid concentrations may be due to the small group size. There is a tendency to difference and the changes 
are similar to the one observed on day 1 . These findings are in agreement with findings in healthy volunteers: structured triglyceride emulsions caused lower plasma levels of medium-chain free fatty acids and were cleared faster from the blood than emulsions of a physical mixture of medium- and long-chain triglycerides. 10,11

One could speculate on the mechanism by which the structured lipids improve nitrogen balance. The present study demonstrates a faster clearance of structured lipid compared with the emulsion of mediumand long-chain triglycerides. A study in patients comparing the administration of structured triglycerides and long-chain triglycerides demonstrated a faster clearance and an increase in oxidation rate. ${ }^{16}$ This suggests that structured triglycerides are hydrolyzed and oxidized faster.

This is also suggested by the study of Hultin et al. ${ }^{26}$ The hypothesis in this study was that the positional specificity of lipoprotein lipase should lead to differences in metabolism in metabolism of a long-chain fatty acid in the 2-position of triglycerides, compared with one in the 1,3-position. In vitro experiments have shown that in physical mixtures of long-chain and medium-chain triglycerides, the medium-chain triglycerides are hydrolyzed more rapidly, and the remnant particles become enriched in long-chain triglycerides. ${ }^{27}$ Also, the lipoprotein lipase shows specificity to the 1,3-position, resulting in the products fatty acids and 2-monoglycerides. ${ }^{28}$ Monoglycerides recirculate in the plasma to a lesser degree than fatty acids. ${ }^{29}$ According (6) these observations, a structured triglyceride with a medium chain fatty acid-long chain fatty acid-medium chain fatty acid (MLM) structure should be cloared faster, compared with long-chain triglycerides or a physical mixture of long-chain and medium-chain triglycerides. In this study a MLM structured triglycoride was compared with long-chain triglycerides and a physical mixture. The hypothesis was confirmed. The clearance and oxidation of MLM was faster, but oxidation was faster only in fasted unanesthetized rats. The differonces were small. In this study no other molecular forms of structured triglycerides were studied. The study of Fultin explains only in part the observations in our study.

The second question is the relation between the faster clearance and oxidation of structured triglycerides and the influence on the protein metabolism. This faster oxidation of structured triglycerides indicates a preference for structured triglycerides as a energy source. Structured triglycerides influences the nitrogen metabolism and may induce a protein saving effect by an increase in protein production or decrease in protein breakdown. Animal studies demonstrated an increase in hepatic protein synthesis ${ }^{13,14}$ and decreased leucine oxidation. ${ }^{12,14}$. The process which regulates this change in protein metabolism is not yet fully studied.

In conclusion, in moderately catabolic patients the parenteral structured triglyceride emulsion improves the nitrogen balance and is cleared faster from blood, compared with a physical mixture of medium- and long-chain triglycerides.

\section{REFERENCES}

1. Campos ACL, Meguid MM: A critical appraisal of the usefulness of perioperative nutritional support. Am J Clin Nutr 55:117-130, 1992

2. The Veterans Affairs TPN Cooperative Study Group: Perioperative TPN in surgical patients. $N$ Engl J Med 325:525-532, 1991

3. Fan S, Lo C, Lai ECS, et al: Perioperative nutritional support in patients undergoing hepatectomy for hepatocellular carcinoma. N Engl J Med 331:1547-1552, 1994

4. Jiang Z, Zhang S, Wang X, et al: A comparison of medium-chain and long-chain triglycerides in surgical patients. Ann Surg 217: 173-184, 1993

5. Dennison AR, Ball M, Hands LJ, et al: TPN using conventional and medium chain triglycerides: Effect on liver function tests, complement, and nitrogen balance. JPEN 12:15-19, 1988

6. Johnson RC, Cotter R: Metabolism of medium-chain triglyceride lipid emulsion. Nutr Int 2:150-158, 1986

7. Kolb S, Sailer D: Effect of fat emulsions containing mediumchain triglycerides and glucose on ketone body production and excretion. JPEN 8:285-289, 1983

8. Mascioli EA, Randall S, Porter KA, et al: Thermogenesis from intravenous medium-chain triglycerides. JPEN 15:27-31, 1991

9. Miles JM, Cattalini M, Sharbrough FW, et al: Metabolic and neurologic effects of an intravenous medium-chain triglyceride emulsion. JPEN 15:37-41, 1991

10. Flaatten $\mathrm{H}$, Aanderud $\mathrm{L}$, Carneheim $\mathrm{C}$, et al: A randomized, single blind, cross-over study comparing a new structured triglyceride fat emulsion (STG 73403) with Vasolipid [Abstract]. Clinical Nutrition 14 (Suppl 2):58, 1995

11. Thörne A, Nordenström J, Carneheim C, et al: Higher elimination rate of structured triglycerides vs. LCT determined by hypertriglyceridaemic clamp technique [Abstract]. Clinical Nutrition 12 (Suppl 2):3, 1993

12. Maiz A, Yamazaki K, Sobrado J, et al: Protein metabolism during TPN in injured rats using medium-chain triglycerides. Metabolism 33:901-909, 1984

13. Mok KT, Maiz A, Yamazaki $\mathrm{K}$, et al: Structured medium-chain and long-chain triglyceride emulsions are superior to physical mixtures in sparing body protein in the burned rat. Metabolism 33:910-915, 1984

14. Pscheidl E, Richer S, Winzer C, et al: Effects of chemically defined structured lipids on protein metabolism in comparison to physical mixtures in an endotoxin rat model [Abstract]. Clinical Nutrition 13 (Suppl 1):31, 1994

15. Sandström R, Hyltander A, Körner U, et al: Structured triglycerides to postoperative patients: A safety and tolerance study. JPEN 17:153-157, 1993

16. Sandström R, Hyltander A, Körner U, et al: Structured triglycerides were well tolerated and induced increased whole body fat oxidation compared with long-chain triglycerides in postoperative patients. JPEN 19:381-386, 1995

17. Holmberg A, Bergqvist D, Westman B, et al: Cytolcine and fibrinogen response in patients undergoing open abdominal aortic aneurysm surgery. Eur $J$ Vasc Endovasc Surg 17:294-300, 1999

18. Grant JP: Administration of parenteral nutrition solutions. IN Handbook of TPN, Grant JP (ed). W. B. Saunders, Philadelphia, 1992, pp 178-179

19. Knaus WA, Draper EA, Wagner DP, et al: APACHE Ir: A sever. ity of disease classification system. Crit Care Med 13:818-829, 1985

20. Durnin JVGA, Womersley J: Body fat assessed from total body density and its estimation from skinfold thickness: measurements on 481 men and women from 16 to 72 years. Br J Nutr $32: 77-97,1974$

21. De Wijn JF: The role of nutrition in the primary prevention of the complications of atherosclerosis. Ned Tijdschr Geneeskd 119: 492-505, 1975

22. Henry RJ, Cannon DC, Winkelman JW. Nonprotein nitrogenous constituents. IN Clinical Chemistry, Principles and Technics, Di Giorgio J (ed). Harper \& Row, Hagerstown, 1974, pp 556-557

23. Tsuchiya $H$, Hayashi $T$, Sato $M$, et aI: Simultaneous separation 
and sensitive determination of free fatty acids in blood plasma by high-performance liquid chromatography. J Chromatogr 309:4352,1984

24. Hicks JM, Boeckx RL: Glucose and other sugars. IN Pediatric Clinical Chemistry, Glasgow A (ed). W. B. Saunders, Philadelphia, 1984, pp 144-145

25. Jeejeebhoy KN: Bulk or bounce-The object of nutritional support. JPEN 12:539-549, 1988

26. Hultin M, Mullertz A, Zundel MA, et al: Metabolism of emulsions containing medium- and long-chain triglycerides or interesterified triglycerides. J Lipid Res 35:1850-1860, 1994
27. Deckelbaum RJ, Hamilton JA, Mose A, et al: Medium-chain vesus long-chain triacylglycerol emulsion hydrolysis by lipoprotein lipase and hepatic lipase; implications for the mechanism of lipase action. Biochemistry 29:1136-1142, 1990

28. Morley N, Kulksi A: Positional specificity of lipoprotein lipase. J Biol Chem 247:6389-6393, 1972

29. Belfrage $\mathrm{P}$, Elovsson J, Olivecrone $\mathrm{T}$ : Radioactivity in blood and liver partial glycerides, and liver phospholipids after intravenous administration to carbohydrate fed rats of chyle containing double labeled triglycerides. Biochim Biophys Acta 106:45 55, 1965 